\title{
Letter to the editor regarding "Ultrasound features of breast cancer for predicting axillary lymph node metastasis"
}

\author{
Zeng Zeng $^{1}$ (D)
}

Received: 30 September 2018 / Accepted: 24 October 2018 / Published online: 26 October 2018

(c) Springer Science+Business Media, LLC, part of Springer Nature 2018

\section{Dear Editor,}

We read with great interest the article of Guo et al. [1] titled "ultrasound features of breast cancer for predicting axillary lymph node metastasis" in a recent issue of the journal. I have some queries on the article.

Guo et al. used multivariate logistic regression to calculate its OR and gave the result that there was a significant positive relationship between the irregular tumor shape and axillary lymph node metastasis. However, in Table 3 on page 7 , round, oval versus irregular tumor shape, its $[\mathrm{OR}]=2.869$, $[\beta]=0.869$, these results were a contradiction. Because $1 \mathrm{nOR}=\beta$ or $e^{\beta}=\mathrm{OR}$, so when $\mathrm{OR}=0-1, \beta<0$; OR $1-2.718$, $\beta=0-1$; OR $>2.718, \beta>1$. This obvious error should be modified through multivariate analysis.

Another query is, Guo et al. aimed at assess ultrasound's clinical value for predicting axillary lymph node metastasis on breast cancer patients. They evaluated all lesions and recorded tumor size, tumor shape, tumor margin, and other features of tumors. However, they didn't pay attention to features of lymph node; the ratio between long arm and short arm of lymph nodes (long/short arm ratio), maximum cortical thickness of lymph node, the ratio between cortex diameter and medulla diameter of lymph nodes (cortex/ medulla ratio) [2] need to be incorporated if they want to predict axillary lymph node metastasis.

Yours Sincerely.

\section{Compliance with ethical standards}

Conflict of interest The authors declare no conflict of interest.

Ethical approval This article does not contain any studies with animals performed by any of the authors.

\section{References}

1. Guo Q, Dong Z, Zhang L, et al. Ultrasound features of breast cancer for predicting axillary lymph node metastasis. J Ultrasound Med. 2017;37:1354-63.

2. Yu X, Hao X, Wan J, et al. Correlation between ultrasound appearance of small breast cancer and axillary lymph node metastasis. Ultrasound Med Biol. 2018;44(2):342-9.
Zeng Zeng

lois1@163.com

1 Department of Ultrasound, Zhejiang Provincial People's Hospital, Hangzhou, Zhejiang, China 